\title{
Lorentz Violation, Möller Scattering, and Finite Temperature
}

\author{
Alesandro F. Santos $\mathbb{D}^{1}$ and Faqir C. Khanna ${ }^{2,3}$ \\ ${ }^{1}$ Instituto de Física, Universidade Federal de Mato Grosso, 78060-900, Cuiabá, MT, Brazil \\ ${ }^{2}$ Department of Physics and Astronomy, University of Victoria, 3800 Finnerty Road, Victoria, BC, Canada \\ ${ }^{3}$ Physics Department, Theoretical Physics Institute, University of Alberta, Edmonton, AB, Canada \\ Correspondence should be addressed to Alesandro F. Santos; alesandroferreira@fisica.ufmt.br
}

Received 24 January 2018; Revised 8 April 2018; Accepted 23 April 2018; Published 24 May 2018

Academic Editor: Edward Sarkisyan-Grinbaum

Copyright (C) 2018 Alesandro F. Santos and Faqir C. Khanna. This is an open access article distributed under the Creative Commons Attribution License, which permits unrestricted use, distribution, and reproduction in any medium, provided the original work is properly cited. The publication of this article was funded by SCOAP ${ }^{3}$.

Lorentz and CPT symmetries may be violated in new physics that emerges at very high energy scale, that is, at the Planck scale. The differential cross section of the Möller scattering due to Lorentz violation at finite temperature is calculated. Lorentz-violating effects emerge from an interaction vertex due to a CPT-odd nonminimal coupling in the covariant derivative. The finite temperature effects are determined using the Thermo Field Dynamics (TFD) formalism.

\section{Introduction}

The Standard Model (SM) respects the Lorentz and CPT symmetries that are supported by many experiments. Although the SM has achieved a remarkable phenomenological success, there are still unresolved issues. Such problems emerge in a number of different scenarios, most of them related to new physics at the Planck scale $\sim 10^{19} \mathrm{GeV}$. Small Lorentz and CPT violations emerge in theories that unify gravity with quantum mechanics such as string theory [1-3]. There are several other issues that lead to Lorentz violation such as loop quantum gravity $[4,5]$, geometrical effects such as noncommutativity $[6,7]$, torsion [8], and nonmetricity [9]. A theory that allows incorporation of all Lorentz-violating terms together with the SM and general relativity is the Standard Model Extension (SME) $[10,11]$. The SME is an effective field theory that preserves the observer Lorentz symmetry, while the particle Lorentz symmetry is violated. This model is divided into two versions, a minimal extension which has operators with dimensions $d \leq 4$ and a nonminimal version of the SME associated with operators of higher dimensions. Numerous possibilities have been investigated [12-14].

The SME framework is one way to investigate Lorentz and $\mathrm{CPT}$ symmetries violation. A different way is to modify the interaction vertex adding a new nonminimal coupling term to the covariant derivative. This new nonminimal coupling term may be CPT-odd or CPT-even. There are various applications for both terms [15-25]. In this paper, the CPT-odd term is chosen to calculate the Lorentz violation correction to the electron-electron scattering, known as Möller scattering. Corrections to the electron-electron scattering due to Lorentz violation have been studied [26]. In this work, the Z-boson exchange contribution to the amplitude is considered in addition to the electromagnetic interaction. In [26], the minimal version of the SME has been used. Here, the nonminimal CPT-odd term is used to calculate Lorentz violation corrections in the electron-electron scattering. In addition, the finite temperature effects are calculated. The Thermo Field Dynamics (TFD) formalism is used to introduce finite temperature.

TFD formalism is a thermal quantum field theory [2732] where the statistical average of an arbitrary operator is interpreted as the expectation value in a thermal vacuum. The thermal vacuum, defined by $|0(\beta)\rangle$, describes a system in thermal equilibrium, where $\beta=1 / k_{B} T$, with $T$ being the temperature and $k_{B}$ the Boltzmann constant. This formalism depends on the doubling of the original Fock space, composed of the original and a fictitious space (tilde space), using Bogoliubov transformations. The original and tilde space are related by a mapping, tilde conjugation rules. The physical variables are described by nontilde operators. The Bogoliubov transformation is a rotation involving these two spaces. As 
a consequence, the propagator is written in two parts: $T=0$ and $T \neq 0$ components.

This paper is organized as follows. In Section 2, a brief introduction to the TFD formalism is presented. In Section 3, the Lorentz-violating nonminimal coupling is considered. The transition amplitude at finite temperature is determined. The differential cross section for Möller scattering with Lorentz-violating parameter at finite temperature is calculated. In Section 4, some concluding remarks are presented.

\section{Thermo Field Dynamics (TFD)}

Here, an introduction to TFD formalism is presented. It is a real-time formalism of quantum field theory at finite temperature where the thermal average of an observable is given by the vacuum expectation value in an extended Hilbert space. This is achieved by defining a thermal ground state $|0(\beta)\rangle$. Then, the expectation value of an operator $A$ is given as $\langle A\rangle=\langle 0(\beta)|A| 0(\beta)\rangle$. However, two main ingredients are required to achieve this: (1) doubling of degrees of freedom in the Hilbert space and (2) the Bogoliubov transformation. The doubling is defined by the tilde $\left(^{\sim}\right)$ conjugation rules, where the expanded space is $S_{T}=S \otimes \widetilde{S}$, with $S$ being the standard Hilbert space and $\widetilde{S}$ being the fictitious space. The mapping between the tilde $\widetilde{A}_{i}$ and nontilde $A_{i}$ operators is defined by the following tilde (or dual) conjugation rules:

$$
\begin{aligned}
\left(A_{i} A_{j}\right)^{\sim} & =\widetilde{A}_{i} \widetilde{A}_{j}, \\
\left(\widetilde{A}_{i}\right)^{\sim} & =-\xi A_{i}, \\
\left(A_{i}^{\dagger}\right)^{\sim} & =\widetilde{A}_{i}^{\dagger}, \\
\left(c A_{i}+A_{j}\right)^{\sim} & =c^{*} \widetilde{A}_{i}+\widetilde{A}_{j},
\end{aligned}
$$

with $\xi=-1$ for bosons and $\xi=+1$ for fermions. The Bogoliubov transformation is a rotation in the tilde and nontilde variables, thus introducing thermal quantities.

For bosons, Bogoliubov transformations are

$$
\begin{aligned}
& a_{p}=u^{\prime}(\beta) a_{p}(\beta)+v^{\prime}(\beta) \tilde{a}_{p}^{\dagger}(\beta), \\
& a_{p}^{\dagger}=u^{\prime}(\beta) a_{p}^{\dagger}(\beta)+v^{\prime}(\beta) \tilde{a}_{p}(\beta), \\
& \tilde{a}_{p}=u^{\prime}(\beta) \tilde{a}_{p}(\beta)+v^{\prime}(\beta) a_{p}^{\dagger}(\beta), \\
& \tilde{a}_{p}^{\dagger}=u^{\prime}(\beta) \tilde{a}_{p}^{\dagger}(\beta)+v^{\prime}(\beta) a_{p}(\beta),
\end{aligned}
$$

where $u^{\prime}(\beta)=\cosh \theta(\beta)$ and $v^{\prime}(\beta)=\sinh \theta(\beta)$, with $a_{p}^{\dagger}$ and $a_{p}$ being creation and annihilation operators, respectively. Algebraic rules for thermal operators are

$$
\begin{aligned}
& {\left[a(k, \beta), a^{\dagger}(p, \beta)\right]=\delta^{3}(k-p),} \\
& {\left[\tilde{a}(k, \beta), \tilde{a}^{\dagger}(p, \beta)\right]=\delta^{3}(k-p),}
\end{aligned}
$$

and other commutation relations are null.

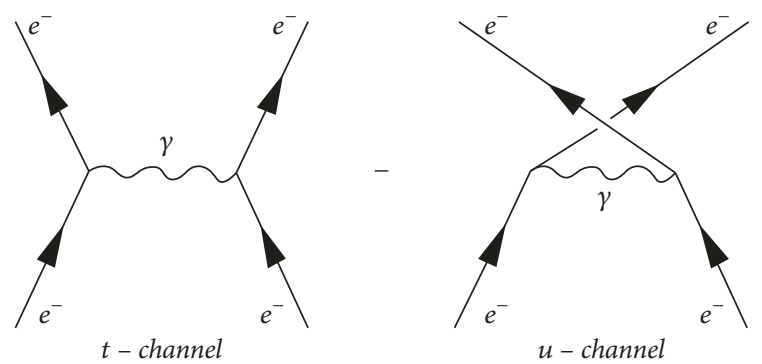

FIGURE 1: Möller scattering.

For fermions, the Bogoliubov transformations are

$$
\begin{aligned}
& c_{p}=\mathrm{u}(\beta) c_{p}(\beta)+\mathrm{v}(\beta) \tilde{c}_{p}^{\dagger}(\beta), \\
& c_{p}^{\dagger}=\mathrm{u}(\beta) c_{p}^{\dagger}(\beta)+\mathrm{v}(\beta) \tilde{c}_{p}(\beta), \\
& \tilde{c}_{p}=\mathrm{u}(\beta) \tilde{c}_{p}(\beta)-\mathrm{v}(\beta) c_{p}^{\dagger}(\beta), \\
& \tilde{c}_{p}^{\dagger}=\mathrm{u}(\beta) \tilde{c}_{p}^{\dagger}(\beta)-\mathrm{v}(\beta) c_{p}(\beta),
\end{aligned}
$$

where $\mathrm{u}(\beta)=\cos \theta(\beta)$ and $\mathrm{v}(\beta)=\sin \theta(\beta)$, with $c_{p}^{\dagger}$ and $c_{p}$ being creation and annihilation operators, respectively. Here, algebraic rules are

$$
\begin{aligned}
& \left\{c(k, \beta), c^{\dagger}(p, \beta)\right\}=\delta^{3}(k-p), \\
& \left\{\widetilde{c}(k, \beta), \tilde{c}^{\dagger}(p, \beta)\right\}=\delta^{3}(k-p),
\end{aligned}
$$

and other anticommutation relations are null.

As an important note, the propagator in TFD formalism is written in two parts: one describes the flat space-time contribution and the other displays the thermal effect.

\section{Lorentz-Violating Corrections to Möller Scattering at Finite Temperature}

Our interest is in calculating the differential cross section for the process, $e^{-}\left(p_{1}\right) e^{-}\left(p_{2}\right) \rightarrow e^{-}\left(p_{3}\right) e^{-}\left(p_{4}\right)$, at finite temperature. This process is represented in Figure 1.

The Lagrangian that describes the Möller scattering is given by

$$
\mathscr{L}=-\frac{1}{4} F_{\mu \nu} F^{\mu \nu}+\bar{\psi}\left(i \gamma^{\mu} D_{\mu}-m\right) \psi-\frac{1}{2 \xi}\left(\partial_{\mu} A^{\mu}\right)^{2} .
$$

To investigate the Lorentz violation in this scattering, an alternative procedure is used. It consists of modifying just the SME interaction part via a nonminimal coupling using the covariant derivative; that is,

$$
D_{\mu}=\partial_{\mu}+i e A_{\mu}+i g b^{\nu} \widetilde{F}_{\mu \nu}
$$

with $e, g$, and $b^{\mu}$ being the electron charge, a coupling constant, and a constant four vector, respectively. $\widetilde{F}_{\mu \nu}=$ $(1 / 2) \epsilon_{\mu \nu \alpha \rho} F^{\alpha \rho}$ is the dual electromagnetic tensor with $\epsilon_{0123}=$ 1 and $F^{\alpha \rho}=\partial^{\alpha} A^{\rho}-\partial^{\rho} A^{\alpha}$. The new interaction breaks the 
Lorentz and CPT symmetries. The interaction Lagrangian is given by

$$
\mathscr{L}_{I}=-e \bar{\psi} \gamma^{\mu} \psi A_{\mu}-g b^{\nu} \bar{\psi} \gamma^{\mu} \psi \partial^{\alpha} A^{\rho} \epsilon_{\mu \nu \alpha \rho} .
$$

The second term represents the new interaction produced by the nonminimal coupling that leads to Lorentz violation, while the first term is the usual QED interaction. These vertices are represented as

$$
\begin{aligned}
\cdot \longrightarrow V^{\mu} & =-i e \gamma^{\mu} \\
\times \longrightarrow g V_{\rho} & =-g b^{\nu} \gamma^{\mu} q^{\alpha} \epsilon_{\mu \nu \alpha \rho},
\end{aligned}
$$

where $q^{\alpha}$ is the momentum operator. The diagrams in Figure 1 represent the usual QED when the vertex $V^{\mu}$ is used and the Lorentz violation corrections are represented by $g V_{\rho}$ vertex. The total contribution is the sum of all diagrams.

In order to calculate the differential cross section for Möller scattering, the transition amplitude,

$$
\mathscr{M}(\beta)=\left\langle f, \beta\left|\hat{S}^{(2)}\right| i, \beta\right\rangle,
$$

is determined. Here, $\widehat{S}^{(2)}$ is the second-order term of the $\widehat{S}$ matrix that is defined as

$$
\begin{aligned}
\widehat{S} & =\sum_{n=0}^{\infty} \frac{(-i)^{n}}{n !} \\
& \cdot \int d x_{1} d x_{2} \cdots d x_{n} \mathbb{\mathbb { L }}\left[\widehat{L}_{I}\left(x_{1}\right) \widehat{L}_{I}\left(x_{2}\right) \cdots \widehat{L}_{I}\left(x_{n}\right)\right],
\end{aligned}
$$

where $\mathbb{T}$ is the time ordering operator and $\widehat{L}_{I}(x)=L_{I}(x)$ $\widetilde{L}_{I}(x)$ describes the interaction. Here, $L_{I}(x)$ and $\widetilde{L}_{I}(x)$ are interaction Lagrangian in usual and tilde Hilbert space, respectively. The thermal states are

$$
\begin{aligned}
|i, \beta\rangle & =c_{p_{1}}^{\dagger}(\beta) d_{p_{2}}^{\dagger}(\beta)|0(\beta)\rangle, \\
|f, \beta\rangle & =c_{p_{3}}(\beta) d_{p_{4}}(\beta)|0(\beta)\rangle,
\end{aligned}
$$

with $\left(c_{p_{j}}^{\dagger}(\beta), d_{p_{j}}^{\dagger}(\beta)\right)$ and $\left(c_{p_{j}}(\beta), d_{p_{j}}(\beta)\right)$ being the creation and annihilation operators, respectively. Then, the transition amplitude becomes

$$
\begin{aligned}
\mathscr{M} & (\beta) \\
= & \frac{(-i)^{2}}{2 !} \int d^{4} x d^{4} y\left\langle f, \beta\left|\left(\mathscr{L}_{I} \mathscr{L}_{I}-\widetilde{\mathscr{L}}_{I} \widetilde{\mathscr{L}}_{I}\right)\right| i, \beta\right\rangle \\
= & \left(\mathscr{M}_{1}(\beta)+\mathscr{M}_{2}(\beta)+\mathscr{M}_{3}(\beta)\right) \\
& -\left(\widetilde{\mathscr{M}}_{1}(\beta)+\widetilde{\mathscr{M}}_{2}(\beta)+\widetilde{\mathscr{M}}_{3}(\beta)\right),
\end{aligned}
$$

where

$$
\begin{gathered}
\mathscr{M}_{1}(\beta)=-\frac{e^{2}}{2} \int d^{4} x d^{4} y\langle f, \\
\left.\beta\left|\bar{\psi}(x) \gamma^{\mu} \psi(x) \bar{\psi}(y) \gamma^{\nu} \psi(y) A_{\mu}(x) A_{\nu}(y)\right| i, \beta\right\rangle, \\
\mathscr{M}_{2}(\beta)=-e g b^{\nu} \epsilon_{\mu \nu \sigma \rho} \int d^{4} x d^{4} y\langle f, \beta| \bar{\psi}(x) \gamma^{\omega} \psi(x) \\
\cdot \bar{\psi}(y) \gamma^{\mu} \psi(y) A_{\omega}(x) \partial^{\sigma} A^{\rho}(y)|i, \beta\rangle, \\
\mathscr{M}_{3}(\beta)=-\frac{1}{2} g^{2} b^{\nu} b^{\rho} \epsilon_{\mu \nu \alpha \sigma} \epsilon_{\omega \rho \delta \gamma} \int d^{4} x d^{4} y\langle f, \beta| \bar{\psi}(x) \\
\cdot \gamma^{\mu} \psi(x) \bar{\psi}(y) \gamma^{\omega} \psi(y) \partial^{\alpha} A^{\sigma}(x) \partial^{\delta} A^{\gamma}(y)|i, \beta\rangle
\end{gathered}
$$

An important note is that the $\mathscr{M}_{3}(\beta)$ contribution will be ignored since it is proportional to the second order of Lorentz-violating parameter. Let us consider the lowest order in this parameter. There are similar equations for the transition amplitude that include tilde operators. The fermion field is written as

$$
\psi(x)=\int d p N_{p}\left[c_{p} u(p) e^{-i p x}+d_{p}^{\dagger} v(p) e^{i p x}\right],
$$

where $c_{p}$ and $d_{p}$ are annihilation operators for electrons and positrons, respectively, $N_{p}$ is the normalization constant, and $u(p)$ and $v(p)$ are Dirac spinors. There are two Feynman diagrams that describe the Möller scattering, the $\mathbf{t}$-channel and the $\mathbf{u}$-channel, as represented in Figure 1. For simplicity, consider

$$
\mathscr{M}_{1}(\beta)=\mathscr{M}_{1}^{\mathbf{t}}(\beta)+\mathscr{M}_{1}^{\mathbf{u}}(\beta),
$$

where $\mathscr{M}_{1}^{\mathbf{t}}(\beta)$ and $\mathscr{M}_{1}^{\mathbf{u}}(\beta)$ are contributions due to the $\mathbf{t}$ channel and the u-channel diagrams, respectively. Then, using (15) and Bogoliubov transformations, we get

$$
\begin{aligned}
& \mathscr{M}_{1}(\beta)=-\frac{e^{2}}{2} N_{p} \int d^{4} x d^{4} y \int d^{4} p\left(u^{2}-v^{2}\right)^{2}\left[\bar{u}\left(p_{2}\right)\right. \\
& \cdot \gamma^{\mu} u\left(p_{1}\right) \bar{u}\left(p_{4}\right) \gamma^{v} u\left(p_{3}\right) e^{i x\left(p_{1}-p_{2}\right)} e^{i y\left(p_{3}-p_{4}\right)}-\bar{u}\left(p_{2}\right) \\
& \left.\cdot \gamma^{\mu} u\left(p_{3}\right) \bar{u}\left(p_{4}\right) \gamma^{\nu} u\left(p_{1}\right) e^{i x\left(p_{2}-p_{3}\right)} e^{i y\left(p_{4}-p_{1}\right)}\right]\langle 0(\beta)| \\
& \cdot \mathbb{T} A_{\mu}(x) A_{\nu}(y)|0(\beta)\rangle .
\end{aligned}
$$

The photon propagator at finite temperature is defined as

$$
\begin{aligned}
& \left\langle 0(\beta)\left|\mathbb{T} A_{\mu}(x) A_{\nu}(y)\right| 0(\beta)\right\rangle \\
& \quad=i \int \frac{d^{4} q}{(2 \pi)^{4}} e^{-i q(x-y)} \Delta_{\mu \nu}(q, \beta),
\end{aligned}
$$

with $\Delta_{\mu \nu}(q, \beta) \equiv \Delta_{\mu \nu}^{(0)}(q)+\Delta_{\mu \nu}^{(\beta)}(q)$, where $\Delta_{\mu \nu}^{(0)}(q)$ and $\Delta_{\mu \nu}^{(\beta)}(q)$ are zero and finite temperature components, respectively. Explicitly, these are

$$
\begin{aligned}
\Delta_{\mu \nu}^{(0)}(q) & =\frac{\eta_{\mu \nu}}{q^{2}}\left(\begin{array}{cc}
1 & 0 \\
0 & -1
\end{array}\right), \\
\Delta_{\mu \nu}^{(\beta)}(q) & =-\frac{2 \pi i \delta\left(q^{2}\right)}{e^{\beta q_{0}}-1}\left(\begin{array}{cc}
1 & e^{\beta q_{0} / 2} \\
e^{\beta q_{0} / 2} & 1
\end{array}\right) \eta_{\mu \nu} .
\end{aligned}
$$


Taking $\mathrm{u}(\beta)=\cos \theta(\beta)$ and $\mathrm{v}(\beta)=\sin \theta(\beta)$, we get $\left(\mathrm{u}^{2}-\right.$ $\left.v^{2}\right)^{2}=\tanh ^{2}\left(\beta\left|q_{0}\right| / 2\right)$, where $q_{0}=\omega$, and using the definition of the four-dimensional delta function,

$$
\begin{aligned}
& \int d^{4} x d^{4} y e^{-i x\left(p_{1}-p_{3}+q\right)} e^{-i y\left(p_{2}-p_{4}-q\right)} \\
& =\delta^{4}\left(p_{1}-p_{3}+q\right) \delta^{4}\left(p_{2}-p_{4}-q\right),
\end{aligned}
$$

the transition amplitude after carrying out the $q$ integral becomes

$$
\begin{aligned}
& \mathscr{M}_{1}(\beta)=-i e^{2}\left[\bar{u}\left(p_{2}\right) \gamma^{\mu} u\left(p_{1}\right) \Delta^{\prime}\left(p_{1}-p_{2}, \beta\right) \bar{u}\left(p_{4}\right)\right. \\
& \cdot \gamma_{\mu} u\left(p_{3}\right) \tanh ^{2}\left(\frac{\beta\left|\left(p_{1}-p_{2}\right)_{0}\right|}{2}\right)-\bar{u}\left(p_{2}\right) \\
& \cdot \gamma^{\nu} u\left(p_{3}\right) \Delta^{\prime}\left(p_{3}-p_{2}, \beta\right) \bar{u}\left(p_{4}\right) \gamma_{\nu} u\left(p_{1}\right) \\
& \left.\cdot \tanh ^{2}\left(\frac{\beta\left|\left(p_{3}-p_{2}\right)_{0}\right|}{2}\right)\right]
\end{aligned}
$$

where $N_{p}=2$ has been used. The propagator is

$$
\Delta_{\mu \nu}^{\prime}(q) \equiv \Delta^{\prime}(q) \eta_{\mu \nu}
$$

with

$$
\begin{aligned}
\Delta^{\prime}(q)= & \frac{1}{q^{2}}\left(\begin{array}{cc}
1 & 0 \\
0 & -1
\end{array}\right) \\
& -\frac{2 \pi i \delta\left(q^{2}\right)}{e^{\beta(q)_{0}}-1}\left(\begin{array}{cc}
1 & e^{\beta(q)_{0} / 2} \\
e^{\beta(q)_{0} / 2} & 1
\end{array}\right) .
\end{aligned}
$$

The remaining delta function, which has the overall fourmomentum conservation, is taken out. In addition, the center of mass is considered so that

$$
\begin{aligned}
& p_{1}=(E, \vec{p}), \\
& p_{2}=(E,-\vec{p}), \\
& p_{3}=\left(E, \overrightarrow{p^{\prime}}\right) \\
& p_{4}=\left(E, \overrightarrow{p^{\prime}}\right),
\end{aligned}
$$

where $|\vec{p}|^{2}=\left|\overrightarrow{p^{\prime}}\right|^{2}=E^{2}, \vec{p} \cdot \overrightarrow{p^{\prime}}=E^{2} \cos \theta$, and $s=(2 E)^{2}=$ $E_{\mathrm{CM}}^{2}$; we get $\left|\left(p_{1}-p_{2}\right)_{0}\right|=\left|\left(p_{3}-p_{2}\right)_{0}\right|=E_{\mathrm{CM}}$. Then,

$$
\begin{aligned}
& \mathscr{M}_{1}(\beta)=-i e^{2}\left[\bar{u}\left(p_{2}\right) \gamma^{\mu} u\left(p_{1}\right) \Delta^{\prime}\left(p_{1}-p_{2}, \beta\right) \bar{u}\left(p_{4}\right)\right. \\
& \cdot \gamma_{\mu} u\left(p_{3}\right)-\bar{u}\left(p_{2}\right) \gamma^{\nu} u\left(p_{3}\right) \Delta^{\prime}\left(p_{3}-p_{2}, \beta\right) \bar{u}\left(p_{4}\right) \\
& \left.\cdot \gamma_{\nu} u\left(p_{1}\right)\right] \tanh ^{2}\left(\frac{\beta E_{\mathrm{CM}}}{2}\right) .
\end{aligned}
$$

In a similar way, the linear term in the Lorentz-violating parameter becomes

$$
\begin{aligned}
& \mathscr{M}_{2}(\beta)=e g b^{v} \epsilon_{\mu v \sigma \rho}\left[\left(p_{1}-p_{2}\right)^{\sigma} \bar{u}\left(p_{2}\right) \gamma^{\rho} u\left(p_{1}\right) \bar{u}\left(p_{4}\right)\right. \\
& \cdot \gamma^{\mu} u\left(p_{3}\right) \Delta^{\prime}\left(p_{1}-p_{2}, \beta\right)-\left(p_{3}-p_{2}\right)^{\sigma} \bar{u}\left(p_{2}\right) \\
& \left.\cdot \gamma^{\rho} u\left(p_{3}\right) \bar{u}\left(p_{4}\right) \gamma^{\mu} u\left(p_{1}\right) \Delta^{\prime}\left(p_{1}+p_{2}\right)\right] \\
& \cdot \tanh ^{2}\left(\frac{\beta E_{\mathrm{CM}}}{2}\right) .
\end{aligned}
$$

The differential cross section is given by

$$
\left(\frac{d \sigma}{d \Omega}\right)_{\beta}=\frac{1}{64 \pi^{2} E_{\mathrm{CM}}^{2}} \cdot \frac{1}{4} \sum_{\text {spins }}|\mathscr{M}(\beta)|^{2} .
$$

Now, an average over the spin of the incoming particles and sum over the spin of the outgoing particles are taken. Then, the square transition amplitude is determined:

$$
|\mathscr{M}(\beta)|^{2}=\left|\mathscr{M}_{1}(\beta)+\mathscr{M}_{2}(\beta)\right|^{2} .
$$

In addition, the relation

$$
\begin{aligned}
& \bar{u}\left(p_{2}\right) \gamma_{\alpha} u\left(p_{1}\right) \bar{u}\left(p_{1}\right) \gamma^{\alpha} u\left(p_{2}\right) \\
& =\operatorname{tr}\left[\gamma_{\alpha} u\left(p_{1}\right) \bar{u}\left(p_{1}\right) \gamma^{\alpha} u\left(p_{2}\right) \bar{u}\left(p_{2}\right)\right]
\end{aligned}
$$

and the completeness relation

$$
\sum u\left(p_{i}\right) \bar{u}\left(p_{i}\right)=\not p_{i}+m
$$

with $i=1,2,3,4$ are used. Now, the electron mass is ignored since the momenta are large; that is, the ultrarelativistic limit $\left(p^{2} \gg m^{2}\right)$ is used.

The differential cross section (see (27)) at finite temperature for a time-like four-vector $b^{v}=(b, 0)$ becomes

$$
\begin{aligned}
& \left(\frac{d \sigma}{d \Omega}\right)_{\beta} \\
& \quad=\frac{1}{256 \pi^{2} s}\left\{e ^ { 4 } \left[\frac{-8 \cos \theta+12 \cos 2 \theta+8 \cos 3 \theta+\cos 4 \theta+115}{2(\cos \theta+1)^{2}}\right.\right. \\
& \left.\left.+C_{1}(\beta)\right]+8 e^{2} g^{2} E^{4} b^{2}\left[\cos 2 \theta+7+C_{2}(\beta)\right]\right\} \tanh ^{2}\left(\frac{\beta E_{\mathrm{CM}}}{2}\right),
\end{aligned}
$$

where

$$
\begin{aligned}
& C_{1}(\beta) \equiv 32 E^{4}\left\{\Delta^{2}\left(p_{1}-p_{2}\right)(\cos \theta+1)^{2}\right. \\
& +\frac{4\left[\Delta\left(p_{1}-p_{2}\right)+\Delta\left(p_{3}-p_{2}\right)\right]^{2} \sin ^{4} \theta}{2} \\
& \left.+4 \Delta^{2}\left(p_{3}-p_{2}\right)\right\} \\
& C_{2}(\beta) \equiv 16 E^{4}\left\{\Delta^{2}\left(p_{1}-p_{2}\right)(\cos 2 \theta+3)\right. \\
& \left.+\frac{4 \Delta^{2}\left(p_{3}-p_{2}\right) \cos ^{4} \theta}{2}\right\}
\end{aligned}
$$


with

$$
\Delta(q)=-\frac{2 \pi i \delta\left(q^{2}\right)}{e^{\beta(q)_{0}}-1}\left(\begin{array}{cc}
1 & e^{\beta(q)_{0} / 2} \\
e^{\beta(q)_{0} / 2} & 1
\end{array}\right)
$$

This result shows that corrections for Möller scattering due to Lorentz violation at finite temperature are altered. Even if the Lorentz symmetry is conserved, there are corrections due to temperature for the usual result for electron-electron scattering.

An important note is that, in the Born approximation, the interaction potential may be calculated using the Fourier transform of the scattering amplitude; that is,

$$
V(r)=\frac{1}{(2 \pi)^{3}} \int \mathscr{M}(\beta) e^{i p \cdot x} d^{3} p
$$

with $\mathscr{M}(\beta)$ being the total scattering amplitude given in (13). In the nonrelativistic limit, some studies have been carried out about electron-electron interaction potential [33-35]. Our result (see (31)) has been obtained in the ultrarelativistic limit, i.e., $p^{2} \gg m^{2}$. However using the nonrelativistic limit (i.e., $\left.p^{2} \ll m^{2}\right)$, in (27), a different cross section at finite temperature and with Lorentz-violating term is obtained. This result may be compared with the nonrelativistic and Lorentz invariant differential cross section at zero temperature that is given as

$$
\begin{aligned}
& \left(\frac{d \sigma}{d \Omega}\right) \\
& \quad=\frac{e^{4}}{4 E^{2}}\left[\left(1-\cos ^{2} \theta\right)+\frac{1}{1+\cos ^{2} \theta}-\frac{1}{(1-\cos \theta)^{2}}\right],
\end{aligned}
$$

where $E$ is the total kinetic energy. In our case, the potential is altered directly by Lorentz violation and temperature effects.

In addition, the result obtained here may be compared with the nonrelativistic result; see (36). Let us consider the following comparisons: (i) $b=0$ and $T \neq 0$-in this case, there are corrections due to temperature effects that become relevant in the high temperature limit since the estimates defined in (34) become large. Then, the dependent temperature part becomes dominant. This result leads to a new motivation: what are the modifications due to Lorentz violation at high temperature for electron-electron scattering? Then, these estimates will give us a reasonable idea of the role of SME parameters at finite temperatures. (ii) $b \neq 0$ and $T=0-$ when the temperature is small, the differential cross section is modified only by Lorentz-violating parameter. Thus, while small, Lorentz-violating terms do not contradict any experimental measurements of the Möller scattering at zero temperature. However, there is a motivation to perform such study at finite temperature. Although Möller scattering at high energy has been investigated in experiments, these are still at zero temperature. There is certainly no investigation at extremely high energy with nonzero temperature that may indicate any role for Lorentz violation.

\section{Conclusion}

Lorentz and CPT symmetries are foundations of SM and general relativity (GR). However, violations of these symmetries could emerge in new physics beyond the SM, at the Planck scale. The SME is an effective field theory that contains SM, GR, and all Lorentz- and CPT-violating operators. In parallel to SME exists an alternative procedure that consists in modifying the interaction part using a nonminimal coupling via covariant derivative. In this paper, the nonminimal coupling term, CPT-odd term, is used to calculate the Lorentzviolating corrections to Möller scattering at finite temperature. Finite temperature effects are introduced using the TFD formalism. Our results show that Lorentz-violating operators at finite temperature contribute to the differential cross section of the electron-electron scattering. This is important since there is no investigation at extremely high energy with nonzero temperature for this scattering. Here, a theoretical study is developed such that relevant effects may arise for processes at very high energies. These results may modify the results that are anticipated for astrophysical processes. In addition, it is shown that the differential scattering cross section for Möller scattering depends on temperature. The cross section also changes with Lorentz violation. Then, this result may be useful to understand the role of Lorentz violation term depending on temperature. The interior of stars is a region where there is a variation of the temperature; then stars may be used to test this result. At present, this result is of theoretical interest and does not provide a direct way to measure an upper limit on the magnitude of the Lorentzviolating nonminimal coupling. Constraints on Lorentz-violating parameter can be obtained if measurements at finite temperature are realized in the future. Although corrections due to squared Lorentz-violating parameters avoid the reach to stronger upper bounds, there are ways to constrain Lorentz violation. The dependence of the cross section on the secondorder Lorentz-violating parameters has been obtained also in other scatterings, such as Bhabha scattering and pair annihilation in the presence of a CPT-odd nonminimal coupling [18, 19] and electron-positron scattering with nonminimal CPTeven coupling term [20]. Good upper bounds for Lorentzviolating parameters have been obtained using experimental data from [36].

\section{Data Availability}

No data were used to support this study.

\section{Conflicts of Interest}

The authors declare that they have no conflicts of interest.

\section{Acknowledgments}

This work by Alesandro F. Santos is supported by CNPq Project 308611/2017-9.

\section{References}

[1] V. A. Kostelecký and S. Samuel, "Spontaneous breaking of Lorentz symmetry in string theory," Physical Review D: Particles, Fields, Gravitation and Cosmology, vol. 39, p. 683, 1989. 
[2] V. A. Kostelecký and R. Potting, "CPT and strings," Theoretical, Phenomenological, and Experimental High Energy Physics. Quantum Field Theory and Statistical Systems, vol. 359, no. 2-3, pp. 545-570, 1991.

[3] V. A. Kostelecký and R. Potting, Physical Review D: Particles, Fields, Gravitation and Cosmology, vol. 51, no. 7, pp. 3923-3935, 1995.

[4] R. Gambini and J. Pullin, "Nonstandard optics from quantum space-time," Physical Review D: Particles, Fields, Gravitation and Cosmology, vol. 59, no. 12, Article ID 124021, 1999.

[5] J. Alfaro, H. A. Morales-Técotl, and L. F. Urrutia, "Loop quantum gravity and light propagation," Physical Review D: Particles, Fields, Gravitation and Cosmology, vol. 65, no. 10, Article ID 103509, 2002.

[6] S. M. Carroll, J. A. Harvey, V. A. Kostelecký, C. D. Lane, and T. Okamoto, "Noncommutative field theory and Lorentz violation," Physical Review Letters, vol. 87, no. 14, 2001.

[7] Z. Guralnik, R. Jackiw, S. Y. Pi, and A. P. Polychronakos, "Testing non-commutative $\mathrm{QED}$, constructing non-commutative MHD," Physics Letters B, vol. 517, no. 3-4, pp. 450-456, 2001.

[8] V. A. Kostelecký, N. Russell, and J. D. Tasson, "Constraints on Torsion from Bounds on Lorentz Violation," Physical Review Letters, vol. 100, no. 11, 2008.

[9] J. Foster, V. A. Kostelecký, and R. Xu, "Constraints on nonmetricity from bounds on Lorentz violation," Physical Review D: Particles, Fields, Gravitation and Cosmology, vol. 95, no. 8, 2017.

[10] D. Colladay and V. A. Kostelecký, "CPT violation and the standard model," Physical Review D: Particles, Fields, Gravitation and Cosmology, vol. 55, article 6760, 1997.

[11] D. Colladay and V. A. Kostelecký, "Lorentz-violating extension of the standard model," Physical Review D: Particles, Fields, Gravitation and Cosmology, vol. 58, Article ID 116002, 1998.

[12] R. Bluhm, S.-H. Fung, and V. A. Kostelecký, "Spontaneous Lorentz and diffeomorphism violation, massive modes, and gravity," Physical Review D: Particles, Fields, Gravitation and Cosmology, vol. 77, no. 6, Article ID 065020, 2008.

[13] V. A. Kostelecký and M. Mewes, "Fermions with Lorentzviolating operators of arbitrary dimension," Physical Review D: Particles, Fields, Gravitation and Cosmology, vol. 88, no. 9, 2013.

[14] Y. Ding and V. A. Kostelecký, "Lorentz-violating spinor electrodynamics and Penning traps," Physical Review D: Particles, Fields, Gravitation and Cosmology, vol. 94, no. 5, 2016.

[15] H. Belich, T. Costa-Soares, M. M. Ferreira Jr., and J. A. HelayëlNeto, "Non-minimal coupling to a Lorentz-violating background and topological implications," The European Physical Journal C, vol. 41, no. 3, pp. 421-426, 2005.

[16] H. Belich, T. Costa-Soares, M. Ferreira, J. Helayël-Neto, and M. Orlando, "A comment on the topological phase for antiparticles in a Lorentz-violating environment," Physics Letters B, vol. 639, no. 6, pp. 675-678, 2006.

[17] H. Belich, L. P. Colatto, T. Costa-Soares, J. A. Helayël-Neto, and M. T. D. Orlando, "Magnetic moment generation from non-minimal couplings in a scenario with Lorentz-symmetry violation," The European Physical Journal C, vol. 62, no. 2, pp. 425-432, 2009.

[18] H. Belich, T. Costa-Soares, M. M. Ferreira Jr., J. A. HelayelNeto, and F. M. O. Mouchereck, "Lorentz-violating corrections on the hydrogen spectrum induced by a nonminimal coupling,"
Physical Review D: Particles, Fields, Gravitation and Cosmology, vol. 74, no. 6, Article ID 065009, 6 pages, 2006.

[19] H. Belich, E. O. Silva, M. M. Ferreira Jr., and M. T. D. Orlando, "Aharonov-Bohm-Casher problem with a nonminimal Lorentz-violating coupling," Physical Review D: Particles, Fields, Gravitation and Cosmology, vol. 83, no. 12, Article ID 125025, 8 pages, 2011.

[20] B. Charneski, M. Gomes, R. V. Maluf, and A. J. da Silva, "Lorentz violation bounds on Bhabha scattering," Physical Review D: Particles, Fields, Gravitation and Cosmology, vol. 86, no. 4, Article ID 045003, 2012.

[21] G. de Brito, J. Guaitolini Junior, D. Kroff, P. Malta, and C. Marques, "Lorentz violation in simple QED processes," Physical Review D: Particles, Fields, Gravitation and Cosmology, vol. 94, no. 5, 2016

[22] R. Casana, M. M. Ferreira, R. V. Maluf, and F. E. dos Santos, “ Effects of a," Physical Review D: Particles, Fields, Gravitation and Cosmology, vol. 86, no. 12, 2012.

[23] R. Casana, M. M. Ferreira, E. Passos, F. E. P. dos Santos, and E. O. Silva, "New CPT-even and Lorentz-violating nonminimal coupling in the Dirac equation," Physical Review D: Particles, Fields, Gravitation and Cosmology, vol. 87, no. 4, Article ID 047701, 2013.

[24] R. Casana, M. M. Ferreira, R. V. Maluf, and F. E. P. dos Santos, "Radiative generation of the CPT-even gauge term of the SME from a dimension-five nonminimal coupling term," Physics Letters B, vol. 726, no. 4-5, pp. 815-819, 2013.

[25] J. B. Araujo, R. Casana, and M. M. Ferreira Jr., "Constraining CPT-even and Lorentz-violating nonminimal couplings with the electron magnetic and electric dipole moments," Physical Review D: Particles, Fields, Gravitation and Cosmology, vol. 92, no. 2, Article ID 025049, 2015.

[26] H. Fu and R. Lehnert, "Møller scattering and Lorentz-violating Z bosons," Physics Letters B, vol. 762, p. 33, 2016.

[27] Y. Takahashi and H. Umezawa, "Thermo field dynamics," Collective Phenomena, vol. 2, pp. 55-80, 1975.

[28] Y. Takahashi and H. Umezawa, "Thermofield dynamics," International Journal of Modern Physics B, vol. 10, no. 13-14, pp. 1755$1805,1996$.

[29] Y. Takahashi, H. Umezawa, and H. Matsumoto, Thermofield Dynamics and Condensed States, North-Holland, Amsterdan, Netherlands, 1982.

[30] H. Umezawa, Advanced Field Theory: Micro, Macro and Thermal Physics, AIP, New York, NY, USA, 1993.

[31] A. E. Santana and F. C. Khanna, "Lie groups and thermal field theory," Physics Letters A, vol. 203, no. 2-3, pp. 68-72, 1995.

[32] A. E. Santana, F. C. Khanna, H. Chu, and Y. Chang, “Thermal Lie groups, classical mechanics, and thermofield dynamics," Annals of Physics, vol. 249, no. 2, pp. 481-498, 1996.

[33] "Low-energy Möller scattering in a Maxwell-Chern-Simons Lorentz-violating model," Physical Review D, vol. 70, Article ID 045013, 2004.

[34] H. Belich, O. M. Del Cima, M. M. Ferreira, and J. A. HelayelNeto, "Electron-Electron Attractive Interaction in MaxwellChern-Simons QED3 at Zero Temperature," International Journal of Modern Physics A, vol. 16, no. 30, pp. 4939-4953, 2001. 
[35] J. Ferreira, "Electron-electron interaction in a Maxwell-ChernSimons model with a purely spacelike Lorentz-violating background," Physical Review D: Particles, Fields, Gravitation and Cosmology, vol. 71, no. 4, Article ID 045003, 9 pages, 2005.

[36] M. Derrick, K. K. Gan, P. Kooijman et al., "Experimental study of the reactions $\mathrm{e}+\mathrm{e}-\rightarrow \mathrm{e}+\mathrm{e}-$ and $\mathrm{e}+\mathrm{e}-\rightarrow \gamma \gamma$ at $29 \mathrm{GeV}$," Physical Review D, p. 3286, 1986. 

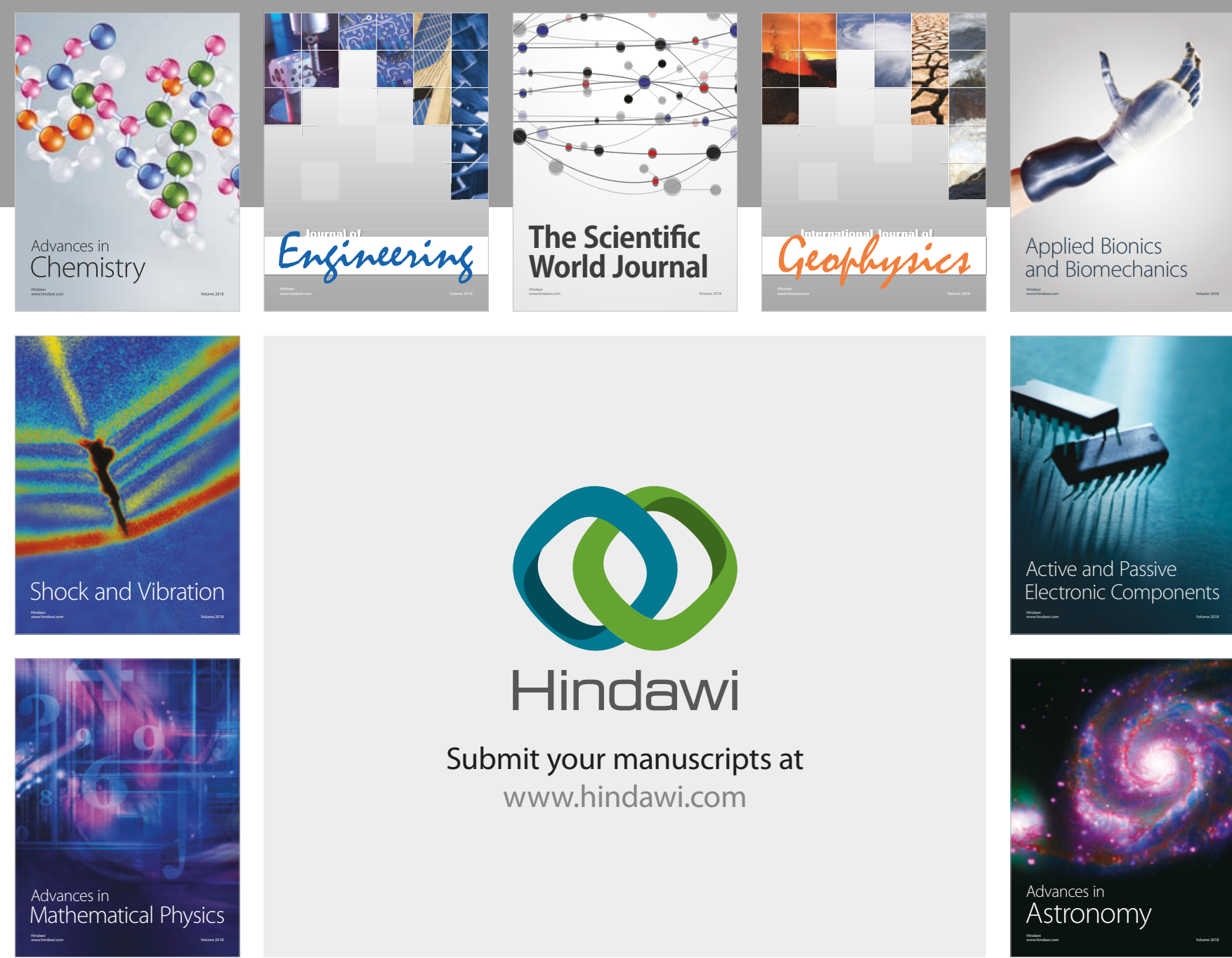

Submit your manuscripts at

www.hindawi.com

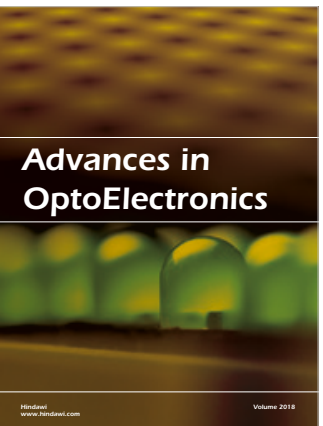

\section{Rotcting Machinery}
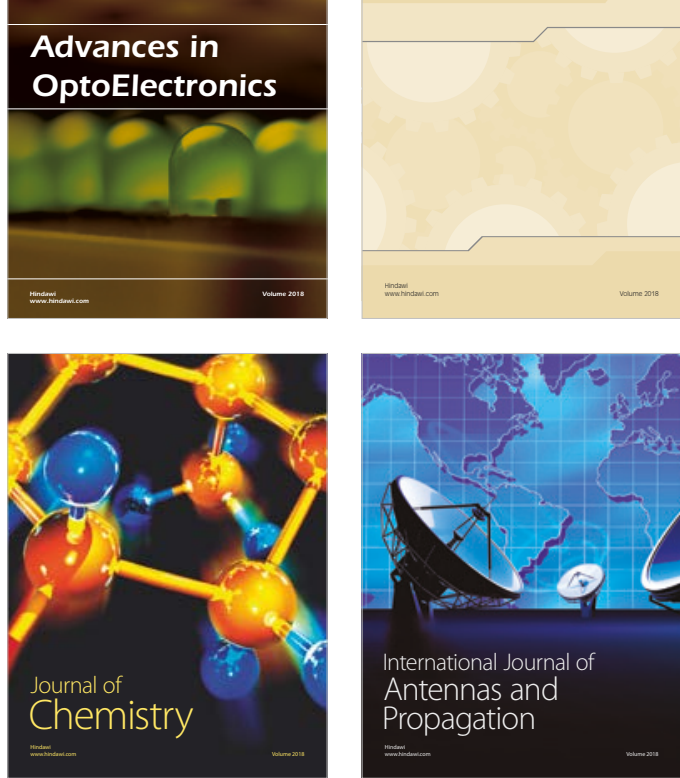

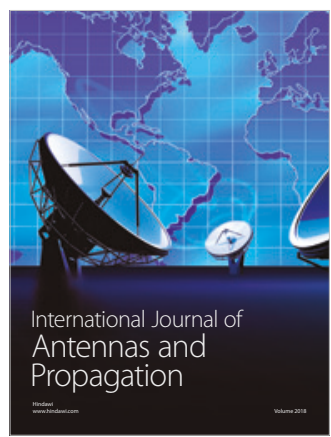

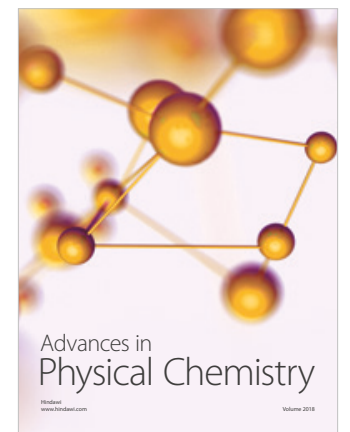

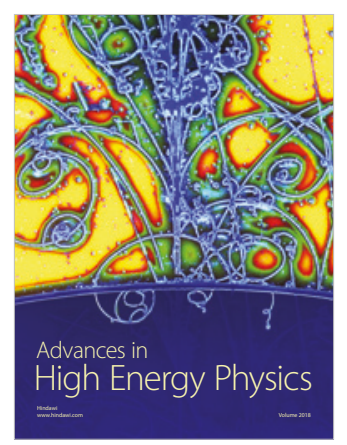

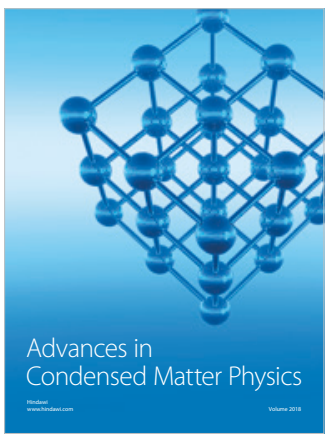

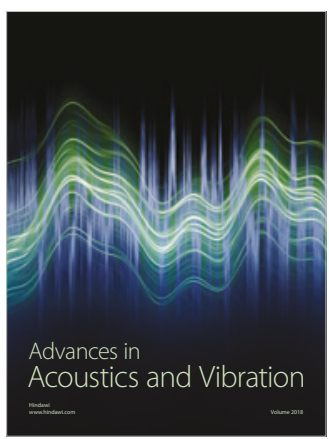

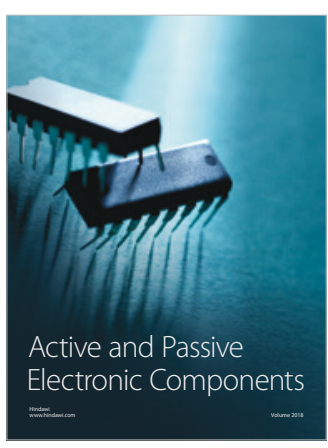
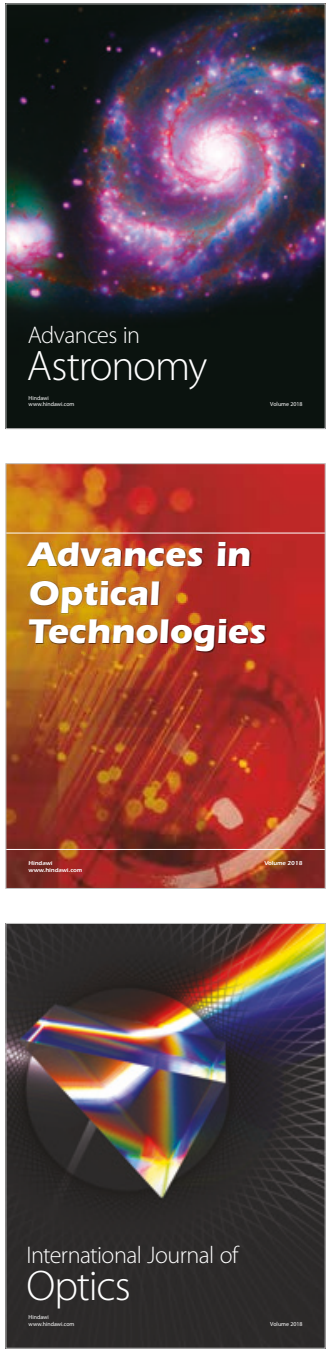